\title{
CK9, AN ANCIENT RAINBOW TROUT CHEMOKINE THAT ATTRACTS AND REGULATES B LYMPHOCYTES AND MACROPHAGES
}

\author{
Carolina Aquilino ${ }^{1}$, Aitor G. Granja ${ }^{1}$, Rosario Castro' ${ }^{1}$, Tiehui Wang ${ }^{2}$, Beatriz Abos ${ }^{1}$, \\ David Parra $^{3}$, Christopher J. Secombes ${ }^{2}$, Carolina Tafalla ${ }^{1}$ \\ ${ }^{1}$ Centro de Investigación en Sanidad Animal (CISA-INIA). Valdeolmos (Madrid), Spain \\ ${ }^{2}$ Scottish Fish Immunology Research Centre, School of Biological Sciences, University of \\ Aberdeen, Aberdeen AB24 2TZ, UK \\ 3 Animal Physiology Unit, Department of Cell Biology, Physiology and Immunology, \\ School of Biosciences, Universitat Autonoma de Barcelona, Cerdanyola del Valles, Spain
}

\begin{abstract}
CK9 is a rainbow trout (Oncorhynchus mykiss) CC chemokine phylogenetically related to mammalian CCL25. Although CK9 is known to be transcriptionally regulated in response to inflammation particularly in mucosal tissues, its functionality has never been revealed. In the current work, we have demonstrated that CK9 has a strong chemotactic capacity for both B cells $\left(\mathrm{IgM}^{+}\right.$and $\left.\operatorname{IgT}^{+}\right)$and macrophages. Along with its chemotactic capacities, CK9 modulated the MHC II turnover of B lymphocytes and up-regulated the phagocytic capacity of both $\mathrm{IgM}^{+}$cells and macrophages. Although CK9 had no lymphoproliferative effects, it increased the survival of $\operatorname{IgT}^{+}$ lymphocytes. Furthermore, we have established that the chemoattractant capacity of CK9 is strongly increased after pre-incubation of leukocytes with a T-independent antigen, whereas B cell receptor (BCR) cross-linking strongly abrogated their capacity to migrate to CK9, indicating that CK9 preferentially attracts B cells at the steady state or under BCR-independent stimulation. These results point to CK9 being a key regulator of B lymphocyte mucosal trafficking in teleost fish, able to modulate innate functions of teleost B lymphocytes and macrophages.
\end{abstract}

\section{KEYWORDS}

Chemokine; CK9; trout; B lymphocytes; macrophages.

$\S$ Corresponding author. Tel.+34 916202300; Fax +34 916202247.

Email address: tafalla@inia.es 